\title{
On a Seminal Paper by Karlin and McGregor
}

Mirta M. CASTRO ${ }^{\dagger}$ and F. Alberto GRÜNBAUM $\ddagger$

${ }^{\dagger}$ Departamento Matemática Aplicada II, Universidad de Sevilla, $c \backslash$ Virgen de África 7, 41011, Sevilla, Spain

E-mail: mirta@us.es

$\ddagger$ Department of Mathematics, University of California, Berkeley, Berkeley, CA 94720 USA

E-mail: grunbaum@math.berkeley.edu

Received August 04, 2012, in final form February 25, 2013; Published online March 02, 2013

http://dx.doi.org/10.3842/SIGMA.2013.020

\begin{abstract}
The use of spectral methods to study birth-and-death processes was pioneered by S. Karlin and J. McGregor. Their expression for the transition probabilities was made explicit by them in a few cases. Here we complete their analysis and indicate a few applications of their very powerful method.
\end{abstract}

Key words: birth-and-death processes; spectral measure

2010 Mathematics Subject Classification: 15A24; 39A99; 47B36; 60J80

\section{Introduction}

\subsection{Preliminaries}

In a paper overflowing with new as well as classical ideas, S. Karlin and J. McGregor [23] considered the birth-and-death process whose one step transition probability matrix $\mathbb{P}$ is given by

$$
\mathbb{P}=\left(\begin{array}{ccccc}
r_{0} & p_{0} & 0 & 0 & \\
q_{1} & r_{1} & p_{1} & 0 & \\
0 & q_{2} & r_{2} & p_{2} & \\
& & \ddots & \ddots & \ddots
\end{array}\right)
$$

where all indicated entries are nonnegative, $p_{0}+r_{0} \leq 1$ and $p_{i}+r_{i}+q_{i} \leq 1, i \geq 1$. These inequalities mean that there is a positive probability of jump to an (ignored) coffin state, or even distinct coffin states for each $i$ such that the corresponding sum is not 1 .

The paper [23] acknowledges and builds on two different earlier approaches: one consists in exploiting the spectral theory of an operator in an appropriate Hilbert space, see [12, 21, 25] and the other one relies on a hands-on study of sample path properties, see [13, 18, 19].

They introduce the polynomials $Q_{j}(x)$ by the conditions $Q_{-1}(0)=0, Q_{0}(x)=1$ and using the notation

$$
Q(x)=\left(\begin{array}{c}
Q_{0}(x) \\
Q_{1}(x) \\
\vdots
\end{array}\right)
$$

they enforce the recursion relation

$$
\mathbb{P} Q(x)=x Q(x)
$$


to argue the existence of a unique measure $\psi(d x)$ supported in $[-1,1]$ such that

$$
\pi_{j} \int_{-1}^{1} Q_{i}(x) Q_{j}(x) \psi(d x)=\delta_{i j}
$$

Then they obtain what is nowadays called the Karlin-McGregor representation formula

$$
\left(\mathbb{P}^{n}\right)_{i j}=\pi_{j} \int_{-1}^{1} x^{n} Q_{i}(x) Q_{j}(x) \psi(d x)
$$

where the constants $\pi_{j}$ are given by

$$
\pi_{0}=1, \quad \pi_{j}=\frac{p_{0} p_{1} \cdots p_{j-1}}{q_{1} q_{2} \cdots q_{j}}, \quad j \geq 1
$$

Many general results can be obtained from the representation formula given above; as they remark, this is nothing but the spectral theorem.

After establishing many such results the authors turn their attention to the study of the spectral properties in the case when one has essentially constant transition probabilities, more specifically

$$
\mathbb{P}=\left(\begin{array}{ccccc}
r_{0} & p_{0} & 0 & 0 & \\
q & 0 & p & 0 & \\
0 & q & 0 & p & \\
& & \ddots & \ddots & \ddots
\end{array}\right)
$$

Here the conditions on the parameters $r_{0}, p_{0}, q$ and $p$ are

$$
r_{0} \geq 0, \quad p_{0}>0, \quad r_{0}+p_{0} \leq 1, \quad p, q>0, \quad p+q=1 .
$$

One usually puts $q_{0}=1-r_{0}-p_{0}$ and when $q_{0}>0$ there is a loss of total mass of the initial probability distribution.

If one denotes the spectral measure by $\psi(d x)$ and by $m(z)$ its Stieltjes transform

$$
m(z)=\int_{-1}^{1} \frac{\psi(d x)}{x-z},
$$

there is a classical method to obtain $m(z)$, which we recall now. If $n(z)$ denotes the Stieltjes transform of the measure going along with the "chopped matrix" obtained by deleting the first row and column of $\mathbb{P}$ then one proves that

$$
m(z)=-1 /\left(z-r_{0}+q p_{0} n(z)\right)
$$

The computation of $n(z)$ itself can be made using the same trick; moreover since (in the case of an essentially constant transition matrix) the "twice chopped matrix" is the same as the "chopped matrix" we get that $n(z)$ satisfies $n(z)=-1 /(z+q p n(z))$ or $n(z)^{2} p q+z n(z)+1=0$, which gives, by solving this quadratic equation and observing that $n(z)$ should vanish at infinity, $n(z)=\left(-z+\sqrt{z^{2}-4 p q}\right) /(2 p q)$.

Before proceeding, it is useful to note that the function $n(z)$ is well defined as a single valued function in the complex plane from which we have removed the closed interval $[-2 \sqrt{p q}, 2 \sqrt{p q}]$. As one approaches this cut from above, the function $n(z)$ has a nontrivial imaginary part coming directly from the square root. This square root has positive values on the real axis to the right of the cut and negative values on the real axis to the left of the cut. This basic observation will play an important role at several points below. 
Returning to the expression above one gets for $m(z)$

$$
m(z)=-\frac{1}{z-r_{0}+\frac{p_{0}}{2 p}\left(-z+\sqrt{z^{2}-4 p q}\right)} .
$$

This expression may have zeros in the denominator giving rise to poles in $m(z)$. Observe that none of these zeros can take place for values of $z=x$ in the cut itself. This is, for instance, a consequence of the fact that along the cut the square root term gives a nontrivial imaginary contribution that cannot be cancelled by the other terms in the denominator which are real valued.

A good way to study this function $m(z)$ is to get rid of the square root in the denominator. In this fashion one gets the expression for $m(z)$ already given in [23, Section 2ii], namely

$$
m(z)=\frac{r_{0}-\left(1-\frac{p_{0}}{2 p}\right) z+\frac{p_{0}}{2 p} \sqrt{z^{2}-4 p q}}{\left(1-\frac{p_{0}}{p}\right) z^{2}-2 r_{0}\left(1-\frac{p_{0}}{2 p}\right) z+r_{0}^{2}+\frac{p_{0}^{2} q}{p}} .
$$

There is a classical way of recovering $\psi(d x)$ from $m(z)$ which consists of considering $m(z)$ as meromorphic function defined on an appropriate Riemann surface and inverting this Stieltjes transform. The measure consists of a nice density plus possibly some delta masses with certain weights. The function $m(z)$ is well defined in the same region that makes $n(z)$ single-valued. The continuous part of the measure results from looking at the values of the imaginary part of $m(z)$ on one side of the cut. The discrete masses in $\psi(d x)$ come from the residues at the simple poles of $m(z)$ but a more detailed analysis is given below. Clearly this inversion process requires a bit of careful computation.

There are several references in the literature where the explicit computation of distributions associated to some special classes of Jacobi matrices is handled. One can mention [15] (see also [5]) for the case of periodic coefficients, [1,2] for the case of parameters with constant limits, [9] for asymptotically periodic parameters and [6] in a different general context.

For many other aspects of birth-and-death processes the reader can see [7, 11, 16, 20, 22, 24]. Many of these reference deal with continuous time.

\subsection{The story behind this paper}

In [23] one finds two examples done in some detail. Specifically they deal with the case when $r_{0}=0$, and the case when $p_{0}=p, r_{0}=q$, which insures that the total mass of the initial probability distribution is preserved. For $r_{0}=0$ and $p=p_{0}$, which gives rise to a variant of the Wigner semicircle law, the authors also consider a two-periodic transition probability matrix.

As preparation for a larger project we had planned to complete the analysis given in [23] by considering the case given in (1.2). Fortunately, during this process, Michael Anshelevich pointed us in the direction of some references in a different field, and after appropriate conversions it became clear that a full analyis of the problem posed in [23] was possible by using results in the literature.

The most recent relevant paper is [26] in the context of "free probability" and the reader may also want to consult [4]. Our basic reference is an earlier paper [8] where Stieltjes inversion along the lines indicated above is avoided altogether. The method in [8] consists of using an old method of Christoffel which can be found in the very classical reference [28], and then getting a nice corollary which amounts to using the method of Christoffel in reverse.

In [26] the authors observe that there is a small error in [8] amounting to having normalized the continuous and the discrete parts of $\psi(d x)$ in different fashions. The method used in [26] is 
the method of Stieltjes indicated above. In fairness one should say that in [26] there are several details left out but that the final result, essentially already given in [8], is correct. See also [3].

One should remark that neither of the references [8,26] mention the results in [23]. A look at more recent papers indicates that the results in [23] are not widely known and that several of these cases are being rederived separately. For instance, [27] does not refer to any of the papers $[8,23,26]$ although the author deals with several special cases of the problem considered in these references, such as the Wigner, Marchenko-Pastur and Kesten-McKay measures.

Going back to the problem considered in [23] and by appropriately adapting the results in $[8,26]$ one can see that the general expression for the measure $\psi(d x)$ is given as follows. The continuous part of the measure is given by

$$
\frac{\frac{p_{0}}{2 \pi} \sqrt{4 p q-x^{2}}}{\left(p-p_{0}\right) x^{2}-r_{0}\left(2 p-p_{0}\right) x+p r_{0}^{2}+p_{0}^{2} q} d x, \quad|x| \leq 2 \sqrt{p q} .
$$

To this continuous part one needs to add possibly one or two mass points, and at this point the analysis in $[8,26]$ becomes relevant.

In this paper we do not reproduce the arguments given in $[8,26]$ to obtain the orthogonality measure. Instead we display the results in a "reader friendly" way, we go through the steps showing why certain residues turn out to be positive, and more importantly we exploit the spectral method discussed here to derive some information about "quasi-stationary distributions". This goes beyond the analysis in $[8,23,26]$ and combines this analysis with important work by E. van Doorn and P. Schrijner, see for instance [29, 30]. Some of these results may be hard to obtain unless one uses spectral methods as in this paper and gives a rather nice way to complete the analysis started in [23] a long time ago.

Since our expressions differ slightly from those in [8] and [26] we have checked carefully the internal consistency of our choices, including all normalizations. This required determining the eigenfunctions of the matrix (1.2). Eventually we found out that [7, p. 204] referring to earlier work of Geronimus [14] had already essentially written down these eigenfunctions as

$$
Q_{j}(x)=\left(\frac{q}{p}\right)^{\frac{j}{2}}\left[\frac{2\left(p_{0}-p\right)}{p_{0}} T_{j}\left(x^{*}\right)+\frac{2 p-p_{0}}{p_{0}} U_{j}\left(x^{*}\right)-\frac{r_{0}}{p_{0}} \sqrt{\frac{p}{q}} U_{j-1}\left(x^{*}\right)\right],
$$

where $T_{j}$ and $U_{j}$ are the Chebyshev polynomials of the first and second kind, and $x^{*}=x /(2 \sqrt{p q})$.

When the measure is made up of the continuous part given above plus the possible contributions coming from the residues at the simple poles of $m(z)$, the squared norms of these polynomials are as in (1.1):

$$
\pi_{0}=1, \quad \pi_{j}=\frac{p_{0} p^{j-1}}{q^{j}}, \quad j \geq 1 .
$$

\section{Beyond Karlin and MacGregor}

\subsection{A single pole}

The denominator of $m(z)$ has a single root exactly when $p_{0}=p, r_{0} \neq 0$, in which case the root is

$$
x_{1}=r_{0}+p q / r_{0} .
$$

Here is a simple argument showing that the root has to be to the right of the interval $[-2 \sqrt{p q}, 2 \sqrt{p q}]$. Indeed, we must have that $r_{0}+p q / r_{0}$ should be at least $2 \sqrt{p q}$, and the difference of these two quantities can be rewritten as $\left(\sqrt{r_{0}}-\sqrt{p q / r_{0}}\right)^{2}$. 


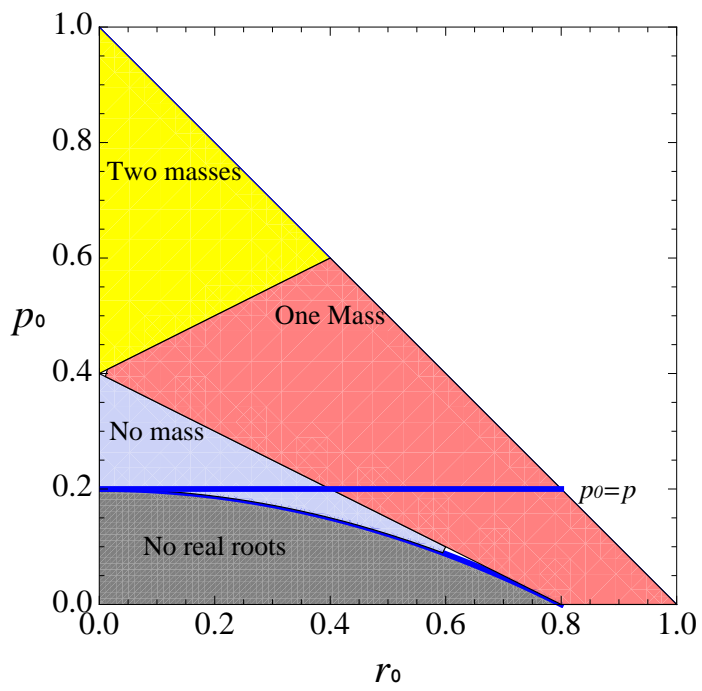

Figure 1. Case $p=0.2$.

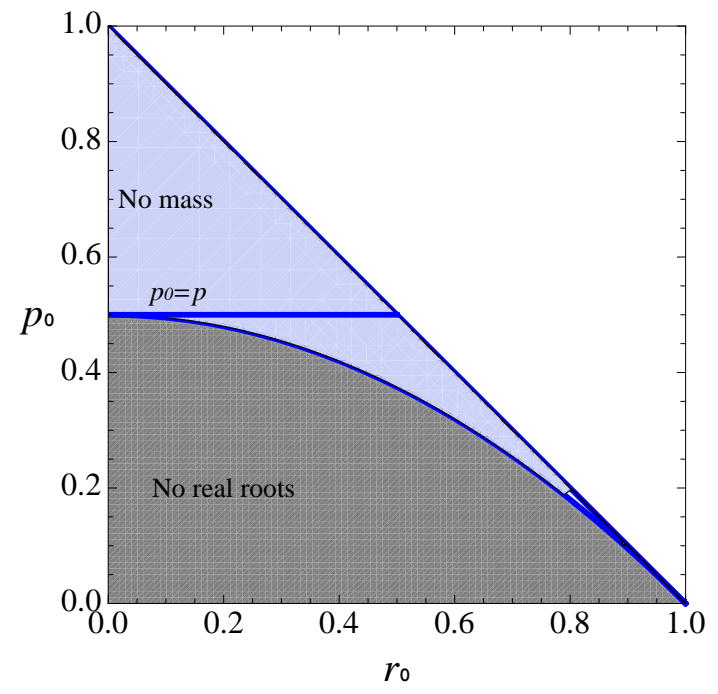

Figure 2. Case $p=0.5$.

In the boundary case $r_{0}=\sqrt{p q}$ the simple root is located at $2 \sqrt{p q}$ and it will turn out that we have no mass.

For $m(z)$ we get the expression

$$
m(z)=\frac{1}{2 r_{0}} \frac{z-2 r_{0}-\sqrt{z^{2}-4 p q}}{z-\left(r_{0}+p q / r_{0}\right)} .
$$

We are interested in the residue of $m(z)$ at (2.1) and this calls for a careful evaluation of the numerator at $z=x_{1}$.

Under the square root we have $\left(r_{0}+p q / r_{0}\right)^{2}-4 p q=\left(r_{0}-p q / r_{0}\right)^{2}$. Since we are using the determination of the square root function which is positive on the real axis to the right of $2 \sqrt{p q}$ and we have shown that $x_{1} \geq 2 \sqrt{p q}$ we have for the residue the value

$$
\frac{1}{2 r_{0}}\left(r_{0}+\frac{p q}{r_{0}}-2 r_{0}-\left|r_{0}-\frac{p q}{r_{0}}\right|\right)
$$

This can be rewritten as $(1 / 2)\left(-\left(1-p q / r_{0}^{2}\right)-\left|1-p q / r_{0}^{2}\right|\right)$, which gives $-\left(1-p q / r_{0}^{2}\right)_{+}$, where the subindex + denotes, as usual, the positive part. Since the denominator in the definition of $m(z)$ is $x-z$ instead of $z-x$ the weight attached to this root is given by the negative of the residue, namely

$$
\left(1-\frac{p q}{r_{0}^{2}}\right)_{+}
$$

Here and later it is important to note that the presence of zeroes in the denominator of $m(z)$ does not guarantee the presence of a mass at such a zero, since the residue may vanish. But if this residue does not vanish then it is automatically positive as we have seen in the simple case analyzed above. In order to have a mass when $p_{0}=p$ one should have

$$
p<1 / 2 \quad \text { and } \quad r_{0} \in(\sqrt{p q}, 1-p]
$$

see Figs. 1, 2 and 3. 


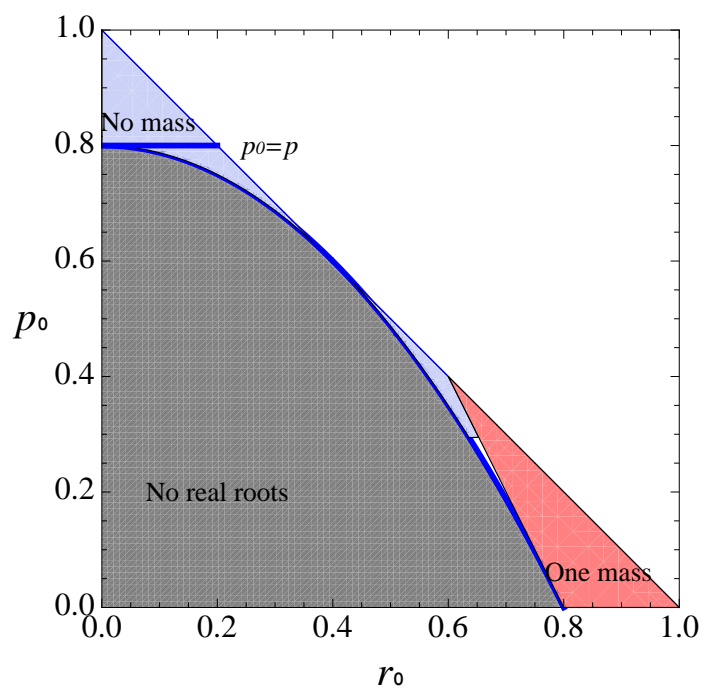

Figure 3. Case $p=0.85$.

\subsection{Two roots}

In the case when the denominator has two different zeros at locations $x_{i}, i=1,2$, an analysis of the residues along the lines of the simpler case above gives for the values of the weights the expressions

$$
\frac{1}{\sqrt{r_{0}^{2}-4 q\left(p-p_{0}\right)}}\left(\frac{q p_{0}^{2}}{p\left|x_{i}-r_{0}\right|}-\left|x_{i}-r_{0}\right|\right)_{+} .
$$

In particular, we see that to have a mass at $x_{i}$ we need

$$
r_{0}^{2} \geq 4 q\left(p-p_{0}\right)
$$

as well as

$$
\left(x_{i}-r_{0}\right)^{2}<\frac{q p_{0}^{2}}{p} .
$$

We will describe, in terms of the values of the parameters $r_{0}, p_{0}$ and $p$, the different possibilities according to the number of positive masses we may have. We will show below that we have just one mass exactly when

$$
\begin{aligned}
p & <\frac{1}{2} & \text { and } & 2 p & -\sqrt{\frac{p}{q}} r_{0}<p_{0} \leq 2 p+\sqrt{\frac{p}{q}} r_{0}, \quad \text { or } \\
p & >\frac{1}{2} & \text { and } & p_{0}>2 p-\sqrt{\frac{p}{q}} r_{0}, & r_{0}>\sqrt{p q},
\end{aligned}
$$

see Figs. 1 and 3. Here, the mass point is always located on the right of the cut.

In the same way, we have two masses exactly when

$$
p<\frac{1}{2} \quad \text { and } \quad p_{0}>2 p+\sqrt{\frac{p}{q}} r_{0}
$$

see in particular Fig. 1. In this case the two mass points are located at both sides of the cut in $[-1,1] \backslash[-2 \sqrt{p q}, 2 \sqrt{p q}]$. 
Indeed, we write the explicit expression of each $x_{i}, i=1,2$ :

$$
x_{1,2}=\frac{r_{0}\left(2 p-p_{0}\right) \pm p_{0} \sqrt{r_{0}^{2}-4 q\left(p-p_{0}\right)}}{2\left(p-p_{0}\right)}, \quad p_{0} \neq p .
$$

In order to have a mass at $x_{i}, i=1,2$, considering (2.6), one needs to have

$$
\left|r_{0} \pm \sqrt{r_{0}^{2}-4 q\left(p-p_{0}\right)}\right|<2 \frac{\sqrt{q}}{\sqrt{p}}\left|p-p_{0}\right| .
$$

To analyze carefully inequality (2.10) one has to consider separately the cases $p_{0}<p$ and $p_{0}>p$.

Suppose that $p_{0}<p$. We will show that in this case it is possible to have a mass only at $x_{2}$ when

$$
p_{0}>2 p-\sqrt{\frac{p}{q}} r_{0}
$$

holds true. Indeed, if we suppose that there is a positive mass at $x_{1}$, from (2.10) we can write

$$
r_{0}+\sqrt{r_{0}^{2}-4 q\left(p-p_{0}\right)}<2 \frac{\sqrt{q}}{\sqrt{p}}\left(p-p_{0}\right)
$$

thus one should have

$$
2 \frac{\sqrt{q}}{\sqrt{p}}\left(p-p_{0}\right)>r_{0}
$$

that contradicts (2.5). Indeed, considering the previous inequality and then (2.5) one would have

$$
r_{0}^{2}<\frac{4 q}{p}\left(p-p_{0}\right)^{2} \leq\left(1-\frac{p_{0}}{p}\right) r_{0}^{2},
$$

which is a contradiction.

Analogously, to have a positive mass at $x_{2}$ we write for $(2.10)$

$$
r_{0}-\sqrt{r_{0}^{2}-4 q\left(p-p_{0}\right)}<2 \frac{\sqrt{q}}{\sqrt{p}}\left(p-p_{0}\right),
$$

or equivalently

$$
r_{0}-2 \frac{\sqrt{q}}{\sqrt{p}}\left(p-p_{0}\right)<\sqrt{r_{0}^{2}-4 q\left(p-p_{0}\right)} .
$$

If (2.11) holds true then the expressions in both sides of the previous inequality are nonnegative so we can consider the squared inequality, which happens to be equivalent to (2.11) as well. We remark that when $p_{0}<p(2.11)$ implies $r_{0}>\sqrt{p q}$.

We conclude the analysis of the case $p_{0}<p$ showing that if $x_{2}$ has a positive mass then $x_{2}>2 \sqrt{p q}$. Indeed, the inequality $x_{2}-2 \sqrt{p q}>0$ is equivalent to

$$
r_{0}\left(2 p-p_{0}\right)-4 \sqrt{p q}\left(p-p_{0}\right)>p_{0} \sqrt{r_{0}^{2}-4 q\left(p-p_{0}\right)} .
$$

Using (2.5) one verifies that the left part of the previous inequality is nonnegative thus taking the squares and simplifying conveniently one obtains the equivalent expression

$$
\left(r_{0} \sqrt{p}-\sqrt{q}\left(2 p-p_{0}\right)\right)^{2}>0 .
$$


We point out that in the boundary case $p_{0}=2 p-\sqrt{p / q} r_{0}$ the weight for $x_{2}=2 \sqrt{p q}$ in (2.4) is zero.

For the case $p_{0}>p$, using similar arguments as before, one can see that to have a mass at $x_{1}$ one needs to have

$$
p_{0}>2 p+\sqrt{\frac{p}{q}} r_{0}
$$

Since

$$
p_{0}+r_{0} \leq 1
$$

condition (2.12) implies $p<1 / 2$. Analogously, if (2.11) holds true, there is a positive mass at $x_{2}$. Consequently, in this case one has two masses when (2.9) holds true, and one verifies that $x_{1}<-2 \sqrt{p q}$ and $x_{2}>2 \sqrt{p q}$.

Notice that, in both cases described above, in order to have a mass one should have $p \neq$ $1 / 2$, otherwise we will get a contradiction with condition (2.13) since the parameters must verify (2.11). See Figs. 1, 2 and 3.

One can consider the case when our process is recurrent (see Sections 2 in $[23,29,30]$ ) for characterizations of some probabilistic properties for this kind of model), which holds when

$$
r_{0}+p_{0}=1 \quad \text { and } \quad p \leq \frac{1}{2} .
$$

In this case $z=1$ is a root of the denominator in $m(z)$, and has a mass here only when the process is positive recurrent as noticed in [23, Section 2], which corresponds to $p<1 / 2$ in (2.14). As a very special subcase, one considers $r_{0}=0, p_{0}=1$, corresponding to a reflecting boundary condition at the left most state. Then one has roots of $m(z)$ both at $z=1$ and $z=-1$, having weights only when $p<1 / 2$, as pointed out initially in [23, Section 2] and [22, pp. 108-109].

\subsection{Some useful figures}

Here we have included some useful figures illustrating the different situations that may arise for the roots of the denominator of $m(z)$ in (1.3), discussed above. The nature of the figures depends on the value of a fixed value of the parameter $p$ being less, equal or larger than $1 / 2$. We consider free nonnegative parameters $p_{0}$ and $r_{0}$ satisfying (2.13). As noticed before, in the case of Fig. 1 for $p<1 / 2$ one can see that there may be one or two mass points. These situations are delimited by the lines $p_{0}=2 p-\sqrt{p / q} r_{0}$ and $p_{0}=2 p+\sqrt{p / q} r_{0}$. We point out that in order to have two masses it is necessary that $p_{0}>p$.

For $p=1 / 2$ Fig. 2 shows that we do not have any mass points. For $p>1 / 2$, as Fig. 3 shows, there may be one mass point above the line $p_{0}=2 p-\sqrt{p / q} r_{0}$ and for $p_{0}<p$.

\section{Miscellaneous}

\subsection{Quasi-stationary distribution}

A new notion enters at this point. A quasi-stationary distribution for a Markov chain with an absorbing state (the case of our chain when $q_{0}$ does not vanish) is defined as an initial distribution such that the (conditional) probability of the chain being at state $j$ at time $n$, conditioned on the fact that no absorption has occurred by then, is independent of the time $n$ for all states $j$.

The issue of the existence and/or uniqueness of such distributions can be illuminated by the analysis given above. 
Assume that $q_{0}>0$ so that we have a loss of total mass for any initial probability distribution. Assume further that $p \leq 1 / 2$ so that eventual transition out of the state space is guaranteed. We denote by $p_{-1}(n)$ the probability of having left the state space by time $n$.

One defines a (normalized) probability distribution $\alpha_{i}$ on the nonnegative integers as a quasistationary distribution for our walk if, by denoting with $p_{j}(n)$ the probability that the walk be at state $\mathrm{j}$ at time $\mathrm{n}$ for the random walk with initial distribution $p_{j}(0)=\alpha_{j}$, one gets the relation

$$
\frac{p_{j}(n)}{1-p_{-1}(n)}=\alpha_{j}, \quad \text { for } \quad j=0,1,2, \ldots, \quad n=0,1,2, \ldots
$$

Denote now by $\eta$ the supremum of all points in the support of the orthogonality measure. This can be a point mass to the right of $2 \sqrt{p q}$ or the value $2 \sqrt{p q}$ itself. In both cases one can have $\eta=1$. This value plays a very important role in terms of the existence and uniqueness of quasi-stationary distributions as follows from a more general result in [29, Theorem 4.2].

In the case when $q_{0}>0$ and when absorption at -1 is certain, which holds for $p \leq 1 / 2$ (see [29, Theorem 2.2]), one gets that if $\eta=1$ then there is no quasi-stationary distribution. In our model this situation only occurs for $p=1 / 2$. Indeed, when $p<1 / 2, p_{0}+r_{0}<1$ and $p_{0} \neq p$, $x=1$ is a root of the denominator of $m(z)$ in (1.3) if

$$
p=\frac{p_{0}}{1+p_{0}-r_{0}} .
$$

But in this case one never has a mass at $x=1$, since the positive part of the corresponding residue vanishes in (2.4). When $p<1 / 2, p_{0}+r_{0}<1$ and $p=p_{0}, x=1$ is a root of the denominator of $m(z)$ when $r_{0}=p_{0}$. In this case the value of the weight in (2.2) is equal to zero.

In case that $\eta<1$, which occurs in our model when $p<1 / 2$ and $q_{0}>0$, we have a one parameter family of such distributions parametrized by values of $x$ in the interval $\eta<x<1$.

The actual values of the distributions (which depend on $x$ ) are given by

$$
\alpha_{j}(x)=\pi_{j}(1-x) \frac{Q_{j}(x)}{q_{0}},
$$

where $Q_{j}(x)$ are the eigenfunctions given in (1.4).

\subsection{Ratio limits}

From the very beginning of the work of Karlin and McGregor, involving birth and death processes with discrete or continuous time parameter, one of the most important advantages of the spectral approach was the possibility to study the value of the limit as $n$ grows of quantities such as

$$
P_{i, j}^{n} / P_{k, l}^{n} \text {. }
$$

As [23] points out these are difficult quantities to study and back in the 1930's Doeblin introduced tools to study the behaviour of the ratio of partial sums of these quantities. The work in [23] can thus be seen as getting a Tauberian theorem to go beyond Doeblin's results. In [23, Theorem 2] one finds a proof of the fact that for a recurrent (and aperiodic) walk the limit above is given by the ratio $\pi_{j} / \pi_{l}$. In our case, the walk is aperiodic or non-symmetric when $r_{0} \neq 0$ and recurrent when (2.14) occurs.

Assume that the parameters $r_{0}, p_{0}, p, r_{0} \neq 0$, are such that we have at least one nonzero point mass. From Sections 2.1 and 2.2 we know that we have just one mass when either $p=p_{0}$ and (2.3) holds or $p \neq p_{0}$ along with conditions (2.7) or (2.8) and one finds two masses under condition (2.9). Then one can use a result in [30, Theorem 3.1] to see that the limit exists for all values of the indices $i, j, k, l$ and is equal to

$$
\frac{\pi_{j}}{\pi_{l}} \frac{Q_{i}(\eta) Q_{j}(\eta)}{Q_{k}(\eta) Q_{l}(\eta)}
$$


where $\eta$ is the supremum of all points in the support of the spectral measure, as defined above. One can also see that for any values of the parameters $r_{0}, p_{0}, p, r_{0} \neq 0$, the limits of the subsequences resulting by taking $n$ even and odd respectively in (3.1) do exist. For the symmetric case $r_{0}=0$, these limits where found in [23, Theorem 3] to be equal to (3.2) for $i-j$ and $k-l$ being simultaneously even or odd.

\subsection{The matrix valued case}

We conclude by calling attention to the last section in [23] where the authors consider an extension of their spectral method to the study of a walk on the integers. They show quite explicitly that the orthogonality measure is to be replaced by a matrix valued measure, which they write down. This point is noticed, for instance, in the Math. Reviews note on this paper, MR0100927. In [23] one does not find the corresponding matrix valued orthogonal polynomials or the extension to the matrix valued case of the Karlin-McGregor formula. This was eventually, and independently done much later in [10] and [17].

\section{Acknowledgements}

The authors thank the anonymous referees for their careful reading and useful remarks. The work of the first author is partially supported by MTM2012-36732-C03-03 (Ministerio de Economía y Competitividad), FQM-262, FQM-4643, FQM-7276 (Junta de Andalucía) and Feder Funds (European Union) and that of the second author is partially supported by the Applied Math. Sciences subprogram of the Office of Energy Research, USDOE, under Contract DE-AC03$76 \mathrm{SF} 00098$.

\section{References}

[1] Allaway W.R., On finding the distribution function for an orthogonal polynomial set, Pacific J. Math. 49 (1973), 305-310.

[2] Allaway W.R., The identification of a class of orthogonal polynomial sets, Ph.D. thesis, University of Alberta, Ann Arbor, MI, 1972.

[3] Anshelevich M., Bochner-Pearson-type characterization of the free Meixner class, Adv. in Appl. Math. 46 (2011), 25-45, arXiv:0909.1097.

[4] Anshelevich M., Free martingale polynomials, J. Funct. Anal. 201 (2003), 228-261, math.CO/0112194.

[5] Aptekarev A.I., Asymptotic properties of polynomials orthogonal on a system of contours, and periodic moments of Toda lattices, Math. USSR Sb. 53 (1986), 233-260.

[6] Askey R., Wilson J., Some basic hypergeometric orthogonal polynomials that generalize Jacobi polynomials, Mem. Amer. Math. Soc. 54 (1985), no. 319, iv+55 pages.

[7] Chihara T.S., An introduction to orthogonal polynomials, Mathematics and its Applications, Vol. 13, Gordon and Breach Science Publishers, New York, 1978.

[8] Cohen J.M., Trenholme A.R., Orthogonal polynomials with a constant recursion formula and an application to harmonic analysis, J. Funct. Anal. 59 (1984), 175-184.

[9] Damanik D., Killip R., Simon B., Perturbations of orthogonal polynomials with periodic recursion coefficients, Ann. of Math. (2) 171 (2010), 1931-2010, math.SP/0702388.

[10] Dette H., Reuther B., Studden W.J., Zygmunt M., Matrix measures and random walks with a block tridiagonal transition matrix, SIAM J. Matrix Anal. Appl. 29 (2007), 117-142.

[11] Feller W., An introduction to probability theory and its applications, Vol. 1, John Wiley \& Sons, Inc., New York, 1967.

[12] Feller W., On second order differential operators, Ann. of Math. (2) 61 (1955), 90-105.

[13] Foster F.G., On the stochastic matrices associated with certain queuing processes, Ann. Math. Stat. 24 (1953), 355-360. 
[14] Geronimus J.L., On a set of polynomials, Ann. of Math. (2) 31 (1930), 681-686.

[15] Geronimus J.L., On some equations in finite differences and the corresponding systems of orthogonal polynomials, Zap. Mat. Otdel. Fiz.-Mat. Fak. i Kharkov. Mat. Obshch. 25 (1957), 87-100 (in Russian).

[16] Good I.J., Random motion and analytic continued fractions, Proc. Cambridge Philos. Soc. 54 (1958), $43-47$.

[17] Grünbaum F.A., Random walks and orthogonal polynomials: some challenges, in Probability, geometry and integrable systems, Math. Sci. Res. Inst. Publ., Vol. 55, Cambridge Univ. Press, Cambridge, 2008, 241-260, math.PR/0703375.

[18] Harris T.E., First passage and recurrence distributions, Trans. Amer. Math. Soc. 73 (1952), 471-486.

[19] Hodges Jr. J.L., Rosenblatt M., Recurrence-time moments in random walks, Pacific J. Math. 3 (1953), 127-136.

[20] Ismail M.E.H., Masson D.R., Letessier J., Valent G., Birth and death processes and orthogonal polynomials, in Orthogonal Polynomials (Columbus, OH, 1989), NATO Adv. Sci. Inst. Ser. C Math. Phys. Sci., Vol. 294, Kluwer Acad. Publ., Dordrecht, 1990, 229-255.

[21] Kac M., Random walk and the theory of Brownian motion, Amer. Math. Monthly 54 (1947), 369-391.

[22] Karlin S., A first course in stochastic processes, Academic Press, New York, 1966.

[23] Karlin S., McGregor J., Random walks, Illinois J. Math. 3 (1959), 66-81.

[24] Ledermann W., Reuter G.E.H., Spectral theory for the differential equations of simple birth and death processes, Philos. Trans. Roy. Soc. London. Ser. A. 246 (1954), 321-369.

[25] McKean Jr. H.P., Elementary solutions for certain parabolic partial differential equations, Trans. Amer. Math. Soc. 82 (1956), 519-548.

[26] Saitoh N., Yoshida H., The infinite divisibility and orthogonal polynomials with a constant recursion formula in free probability theory, Probab. Math. Stat. 21 (2001), 159-170.

[27] Sodin S., Random matrices, nonbacktracking walks, and orthogonal polynomials, J. Math. Phys. 48 (2007), 123503, 21 pages, math-ph/0703043.

[28] Szegö G., Orthogonal polynomials, American Mathematical Society, Colloquium Publications, Vol. 23, 4th ed., Amer. Math. Soc., Providence, R.I., 1975.

[29] van Doorn E.A., Schrijner P., Geometric ergodicity and quasi-stationarity in discrete-time birth-death processes, J. Austral. Math. Soc. Ser. B 37 (1995), 121-144.

[30] van Doorn E.A., Schrijner P., Ratio limits and limiting conditional distributions for discrete-time birth-death processes, J. Math. Anal. Appl. 190 (1995), 263-284. 